\title{
Factores pronósticos de mortalidad en pacientes con cáncer de mama HER2 positivo
}

- Pedro Luis Ramos Guette

Correos electrónicos: pramos@oncocareltda, pramos152@yahoo.com

Resumen

Objetivos: el propósito de este estudio fue evaluar la supervivencia global y libre de enfermedad; y los factores pronósticos asociados con estas en mujeres con cáncer de mama HER2 positivo.

Métodos: en este estudio se hizo el análisis de la información de la clínica Oncocare en el período comprendido entre el $1^{\circ}$ de enero de 2003 y el 31 de julio de 2012. Se incluyeron todas las pacientes de 18 o más años con diagnóstico de cáncer de mama HER2 positivo. Se construyeron curvas de supervivencia por el método de Kaplan-Meier, se compararon con el método de rangos logaritmos y se efectuó un análisis de regresión de rangos proporcionales por Cox.

Resultados: un total de 82 pacientes fueron identificadas en el estudio. La tasa de mortalidad de la cohorte fue del 9,76\% y recaídas del $12,2 \%$. La edad media fue de 53,9 años, la mediana de seguimiento fue de 54 meses.

Los estadios clínicos se distribuyeron así: estadio I en el 9,76\%, IIA en el $18,74 \%$, IIB en el $26,83 \%$, IIIA en el $19,69 \%$, IIIB en el $25,61 \%$, IIIC en el $9,76 \%$. Los receptores de estrógenos y de progestágeno son ambos positivos en un $45,12 \%$, los ganglios son positivos en un $42,11 \%$. Las pacientes recibieron quimioterapia neoadyuvante en el $65,85 \%$, del tipo AC en un $61,11 \%$ y le sigue el esquema de ACTH en el $20,37 \%$ y $A C T$ en el $14,81 \%$. Se les administró quimioterapia adyuvante en un $95,12 \%, \mathrm{ACTH}$ en un $75,64 \%$, AC en un $14 \%$ y ACT en un $2,26 \%$. La respuesta a la quimioterapia neoadyuvante fue objetiva en un $83,31 \%$, completa en un $14,81 \%$ y parcial en un $68,52 \%$; se presentaron recaídas en el $12,20 \%$. En el análisis univariado, el único factor asociado con supervivencia libre de enfermedad fue la respuesta a la quimioterapia neoadyuvante, pero en el análisis multivariado no es significativo; los demás factores pronósticos, como el estadio clínico, el receptor hormonal (positivo), la edad y el tamaño tumoral, no se relacionan con tiempos de supervivencia libre de enfermedad y global.

Conclusión: los tiempos de supervivencia libre de enfermedad y global no se asocian con factores como edad, receptores hormonales, estadio clínico, estado ganglionar, tamaño tumoral y las respuestas objetivas a la quimioterapia neoadyuvante.

\section{Características clínicas y demográficas en pacientes con cáncer de pulmón EGFR mutado, Bogotá (Colombia), 2008-2012}

\author{
Pedro Luis Ramos Guette
}

Correos electrónicos: pramos@oncocareltda,pramos152@yahoo.com

\section{Resumen}

Objetivo: la meta de este estudio fue describir las características clínicas y demográficas de los pacientes con cáncer de pulmón EGFR mutado.

Materiales y métodos: se trata de un estudio observacional descriptivo, tipo serie de casos bidireccional; se realiza con la información de la clínica Oncocare en el período comprendido entre el $1^{\circ}$ de diciembre de 2008 y el 31 de diciembre de 2012. Se incluyeron todos los pacientes con diagnóstico de cáncer de pulmón con mutación en el gen EGFR; se hace una descripción de las características clínicas y demográficas con estadística descriptiva.

Resultados: un total de 10 pacientes fueron identificados en el estudio. La edad promedio fue de 51,8 años, la mediana de seguimiento

Palabras clave: cáncer de pulmón, pronóstico, erlotinib. fue de 16,7 meses. La mediana de tiempo a la progresión fue 12,6 meses. El sexo femenino fue predominante con 7 casos, todos fueron adenocarcinoma; y, de estos, 2 con la variante bronquioloalveolar. La presentación clínica inicial fue estadio IIIB: 1 caso, IV: 7 casos y 2 casos de recaídas sistémicas con estadios tempranos iniciales IIA y IIB. Todos los casos fueron en no fumadores; 4 pacientes progresaron, de los cuales 3 fallecieron hasta el fin del seguimiento.

Conclusión: los pacientes con cáncer de pulmón con mutación del gen EGFR tienen unos tiempos de sobrevida prolongados mejores que los pacientes no mutados, que es lo reportado en la literatura; se requiere un esfuerzo nacional para incluir todos los pacientes en tratamiento y tratados en el país para conocer nuestra realidad nacional. 